\title{
Effect of Slip Velocity on Peristaltic Transport of a Magneto-Micropolar Fluid Through a Porous Non-uniform Channel
}

\author{
G. C. Shit - M. Roy
}

Published online: 7 November 2014

(C) Springer India Pvt. Ltd. 2014

\begin{abstract}
In this paper we have investigated the effect of slip velocity on peristaltic transport of a physiological fluid through a porous non-uniform channel under the long wave length and low-Reynolds number assumptions. We analyzed the flow characteristics of incompressible, viscous, electrically conducting micropolar fluid (a non-Newtonian fluid model). The analytical expressions for the axial velocity, microrotation component, stream function as well as the pressure gradient are obtained. The flow phenomena for the pumping characteristics, trapping and reflux are furthermore investigated. The results presented here reveal that the central line velocity decreases with the increase of magnetic field strength as well as the slip parameter. Again the velocity increases with the increase of porous permeability parameter. Moreover the trapping fluid can be eliminated with a considerable extent by the application of an external magnetic field as well as by increasing the slip parameter. Thus the study bears the promise to keep important applications in physiological systems.
\end{abstract}

Keywords Micropolar fluid · Non-uniform channel · Peristaltic transport . Porous Medium · Slip velocity $\cdot$ Magnetic field

\section{Introduction}

Peristaltic transport of a physiological fluid is induced by a propagation of progressive waves of area contraction or expansion along the length of a distensible tube containing fluid. Peristaltic mechanism causes the urine transport from kidney to the bladder, the movement of chyme in the gastrointestinal tract, fluids in the lymphatic vessels, bile from the gallbladder into the duodenum, the movement of spermatozoa in the ductus efferent of the male reproductive tract, the movement of the ovum in the fallopian tube and the circulation of blood in small blood vessels. This mechanism also finds many applications in roller and finger pumps, some bio-mechanical instruments, e.g. heart-lung machine, blood pump machine and dialysis

G. C. Shit $(\bowtie) \cdot$ M. Roy

Department of Mathematics, Jadavpur University, Kolkata 700032, India

e-mail: gopal_iitkgp@yahoo.co.in 
machine. Thus, peristaltic transport has been the recent studies of many researchers/scientists owing to the above mentioned applications in bio-mechanical engineering and bio-medical technology.

First attempt was made by Latham [16] to study the mechanism of peristaltic transport and later on several theoretical and experimental studies [12,37] have been investigated to understand the clear idea of peristaltic action in different conditions. Many authors [21,25,28,36] studied on the peristaltic transport of particle-fluid suspension model under different situations. Misra and Pandey [26] presented a mathematical model of peristaltic transport of blood in small vessels. The peristaltic transport of a Power-law fluid in the male reproductive tract was examined by Srivastava and Srivastava [35]. A theoretical study of peristaltic flow in a non-uniform small blood vessel has been made by Lee and Fung [17]. Mishra and Rao [23] and Rao and Mishra [30] presented two important results on peristaltic transport in an asymmetric channel by taking two different fluid models, wherein they pointed out that the pumping against pressure rise, trapping and reflux layer exists only when cross-section of the channel varies. The peristaltic waves on the walls with same amplitude propagating in phase produce zero flux rate as the channel cross-section remains same throughout. Eytan and Elad [9] have developed a mathematical model of wall-induced Intra-uterine fluid transport within a two dimensional asymmetric channel of different amplitude and phase.

Because of the complex rheology of biological fluids, it behaves as a suspensions of deformable or rigid particles in a Newtonian viscous fluid. For example, blood is a suspension of red cells, white cells, platelets in plasma and cervical mucus is a suspension of macro molecules in water like liquids. However, Eringen [8] initiated the concept of non-Newtonian fluid known as micropolar fluid to characterize the suspensions of neutrally buoyant rigid particles in a viscous fluid. In the micropolar theory a part of classical velocity field, the microrotation vector and the gyration parameter are introduced to investigate the kinematics of microrotation. Keeping this in mind the behaviour of micropolar fluid have been studied by several investigators $[1,2,18,20,31,32,39]$ by considering the peristaltic transport of micropolar fluid in different situations. The study of MHD flow of an electrically conducting fluid on the peristaltic transport has became a subject of growing interest for researchers and clinicians, because of its useful applications in magnetic resonance imaging (MRI) and pumping of blood. Vishnyakov et al. [38] investigated the peristaltic flow of electrically conducting fluid in the presence of a traverse magnetic field. Jafrin and Shapiro [13] studied peristaltic pumping with long wave length and low Reynolds number approximations.

Since the vessel wall, human lungs and gallbladder are surrounded by flattened endothelial cell, it may consider as a porous medium. In many biomechanical studies, porosity of the media has significant role on the transportation of fluids. El-Shehawey and Husseny [7] studied the peristaltic transport through a porous medium with effect of porous boundaries by taking into consideration of symmetric channel with flexible wall. Ellahi et al. [6] have examined the peristaltic flow of a Carreau fluid in a rectangular duct. They solved the problem analytically by using homotopy perturbation and eigenfunction expansion method under long wave length and low Reynolds number assumptions. Mekheimer and Al-Arabi [22] put forwarded an idea about the non-linear peristaltic transport of MHD flow through porous medium. Khan et al. [14] made an investigation on peristaltic transport of a Jeffrey fluid with variable viscosity through a porous medium in an asymmetric channel. Rao and Mishra [24] investigated the peristaltic transport of Power law fluid in a porous tube. Misra et al. [27] presented an analytical approach to study the peristaltic transport of a physiological fluid in an asymmetric porous channel in presence of an external magnetic field. However, Kothandapani and Srinivas [15] considered the non-linear peristaltic transport of Newtonian fluid in a porous inclined asymmetric channel. Shit and Roy [33] examined the effects of magnetic field on inclined peristaltic 


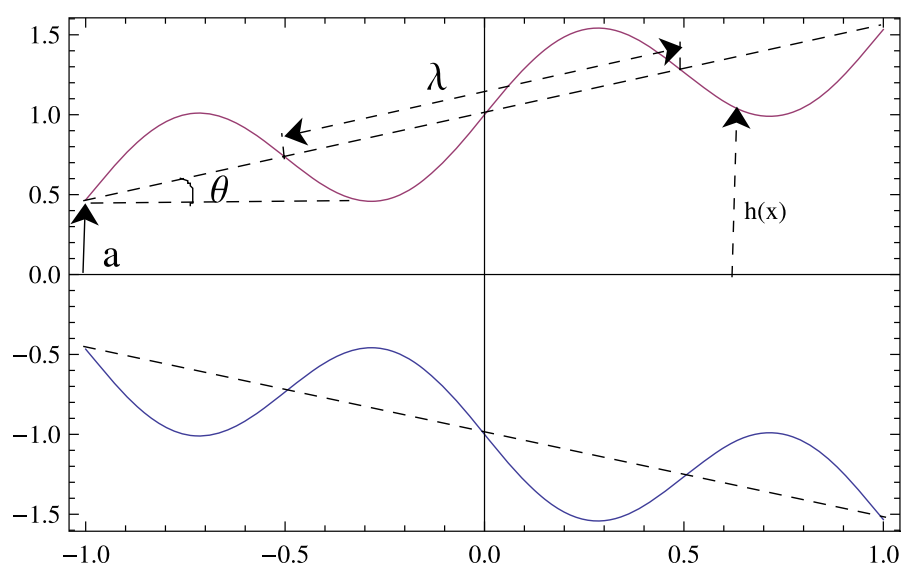

Fig. 1 A Physical sketch of the problem

flow of Couple stress fluid. Hall effect on peristaltic flow of Maxwell fluid in a porous medium have been examined by Hayat et al. [10]. Sobh and Mady [34] have made an important observation on the peristaltic transport through porous medium in a non-uniform channel by taking into account the Newtonian fluid model. Hayat et al. [11] studied the influence of slip velocity on the peristaltic motion of a third order fluid in an asymmetric channel. Chaube et al. [3] carried out the effect of slip velocity on the peristaltic flow in a porous medium by considering the micropolar fluid model. Ellahi [4] and Ellahi et al. [5] have investigated the effects of nonlinear slip boundary condition on the nonlinear flows of various non-Newtonian fluid models.

Owing to the above mentioned observations, we have undertaken here a study that concerns peristaltic flow through a non-uniform porous channel in the presence of an external magnetic field. The micropolar fluid model has been considered in order to examine the effect of microrotaion of microparticles. Moreover, the effects of slip velocity have been rigorously studied in the present investigation. Therefore, the main findings of the present study will serve as a reasonably good estimate of various parameters in a pathological state.

\section{Mathematical Formulation}

Let us consider the flow of a unsteady, incompressible, viscous and electrically conducting micro-polar fluid through a non-uniform porous channel of uniform thickness under the action of an external magnetic field. Let $Y^{\prime}= \pm h^{\prime}\left(X^{\prime}, t^{\prime}\right)$ denote the upper and lower wall of the channel is considered to be induced by a sinusoidal wave train propagating with a wave speed $c$ along the length of the channel wall (cf. Fig. 1), such that

$$
h^{\prime}\left(X^{\prime}, t^{\prime}\right)=a+\tan (\theta)\left(X^{\prime}-c t^{\prime}\right)+b \sin \left(\frac{2 \pi}{\lambda}\left(X^{\prime}-c t^{\prime}\right)\right),
$$

where $a$ is the half width of the channel at the inlet, $\lambda$ is the wave length, $b$ is the amplitude of wave, $\theta$ is the angle between the axis of the channel and the walls, $X^{\prime}$ and $Y^{\prime}$ represent the rectangular co-ordinates with $X^{\prime}$ measured the axis of the channel and $Y^{\prime}$ the traverse axis perpendicular to $X^{\prime}$. 
The system is stressed by an external transverse uniform constant magnetic field of strength $B_{0}$ and hence total magnetic field induction vector is $\mathrm{B}\left(0, B_{0}, 0\right)$, where the induced magnetic field have been neglected due to the assumption of weak electrical conductivity.

The equations of motion for unsteady flow through porous medium of an incompressible magneto-micro-polar fluid with externally imposed magnetic field by neglecting the body couples are,

$$
\begin{aligned}
\nabla \cdot \mathbf{q}^{\prime}= & 0, \\
\rho\left(\frac{\partial \mathbf{q}^{\prime}}{\partial t^{\prime}}+\left(\mathbf{q}^{\prime} \cdot \nabla\right) \mathbf{q}^{\prime}\right)=- & \nabla p^{\prime}+(\eta+k) \nabla^{2} \mathbf{q}^{\prime}+k\left(\nabla \times \boldsymbol{\Omega}^{\prime}\right)+J \times B \\
& \quad-\frac{(\eta+k)}{k_{p}} \mathbf{q}^{\prime}, \\
\rho j^{\prime}\left(\frac{\partial \boldsymbol{\Omega}^{\prime}}{\partial t^{\prime}}+\left(\mathbf{q}^{\prime} \cdot \nabla\right) \boldsymbol{\Omega}^{\prime}\right)=- & 2 k \boldsymbol{\Omega}^{\prime}+k \boldsymbol{\nabla} \times \mathbf{q}^{\prime}-\gamma\left(\nabla \times \nabla \times \boldsymbol{\Omega}^{\prime}\right) \\
& +(\alpha+\beta+\gamma) \nabla\left(\nabla \cdot \boldsymbol{\Omega}^{\prime}\right),
\end{aligned}
$$

along with the generalized Ohm's law $J=\sigma\left(E+\mathbf{q}^{\prime} \times B\right)$.

where $\mathbf{q}^{\prime}=\left(U^{\prime}, V^{\prime}, 0\right)$ be the velocity vector, $\boldsymbol{\Omega}^{\prime}=\left(0,0, W^{\prime}\right)$ the microrotation vector, $p^{\prime}$ the total fluid pressure, $\rho$ the fluid density, $j^{\prime}$ the micro-gyration parameter, J current density vector, $\sigma$ electrical conductivity of fluid and $E$ is the electric field vector. Also the material constants (or viscosity coefficients of the micro-polar fluid ) $\eta, k, \alpha, \beta$ and $\gamma$ are satisfied the following inequalities $(2 \eta+k) \geq 0, k \geq 0,(3 \alpha+\beta+\gamma) \geq 0, \gamma \geq|\beta|$.

Let us consider a wave frame $\left(x^{\prime}, y^{\prime}\right)$ moving with the velocity $c$ away from the fixed frame $\left(X^{\prime}, Y^{\prime}\right)$ are connected by the following transformations

$$
x^{\prime}=X^{\prime}-c t^{\prime}, y^{\prime}=Y^{\prime}, u^{\prime}=U^{\prime}-c, v^{\prime}=V^{\prime}, w^{\prime}=W^{\prime},
$$

where $\left(U^{\prime}, V^{\prime}\right)$ and $\left(u^{\prime}, v^{\prime}\right)$ are the components of velocity in the fixed frame and wave frame of reference respectively.

Now, we introduce the following dimensionless variables

$$
\begin{aligned}
& x=\frac{x^{\prime}}{\lambda}, y=\frac{y^{\prime}}{a}, u=\frac{u^{\prime}}{c}, v=\frac{\lambda v^{\prime}}{a c}, w=\frac{a w^{\prime}}{c}, h(x)=\frac{h^{\prime}\left(x^{\prime}\right)}{a}, p=\frac{a^{2} p^{\prime}\left(x^{\prime}\right)}{\lambda \eta c}, \\
& t=\frac{c t^{\prime}}{\lambda}, j=\frac{j^{\prime}}{a^{2}} .
\end{aligned}
$$

Using the transformations defined in (5) and the dimensionless variables (6) into the equations (3) and (4) and with the use of Ohm's law under the assumption of low electric field intensity yields

$$
\begin{aligned}
R_{e} \delta\left((u+1) \frac{\partial u}{\partial x}+v \frac{\partial u}{\partial y}\right)= & -\frac{\partial p}{\partial x}+(1+K)\left(\delta^{2} \frac{\partial^{2} u}{\partial x^{2}}+\frac{\partial^{2} u}{\partial y^{2}}\right)+K \frac{\partial w}{\partial y} \\
& -\left(H_{a}^{2}+\frac{(1+K)}{K_{p}}\right)(u+1), \\
R_{e} \delta^{3}\left((u+1) \frac{\partial v}{\partial x}+v \frac{\partial v}{\partial y}\right)= & -\frac{\partial p}{\partial y}+(1+K) \delta^{2}\left(\delta^{2} \frac{\partial^{2} v}{\partial x^{2}}+\frac{\partial^{2} v}{\partial y^{2}}\right)-K \delta^{2} \frac{\partial w}{\partial x} \\
& -\delta^{2} \frac{(1+K)}{K_{p}} v, \\
R_{e} \delta j\left((u+1) \frac{\partial w}{\partial x}+v \frac{\partial w}{\partial y}\right)= & -2 K w+K\left(\delta^{2} \frac{\partial v}{\partial x}-\frac{\partial u}{\partial y}\right)+M\left(\delta^{2} \frac{\partial^{2} w}{\partial x^{2}}+\frac{\partial^{2} w}{\partial y^{2}}\right) .
\end{aligned}
$$


Now, we define the following dimensionless parameters that appear in the Eqs. (7)-(9) as $R_{e}=\frac{c a \rho}{\eta}$ be the Reynolds number, $\delta=\frac{a}{\lambda}$ the wave number, $K=\frac{k}{\eta}(0 \leq K \leq 1)$ the ratio of viscosity parameter, $M=\frac{\gamma}{\eta a^{2}}$ the material constant or micropolar parameter, $H_{a}=B_{0} a \sqrt{\frac{\sigma}{\eta}}$ the Hartmann number and $K_{p}=\frac{k_{p}}{a^{2}}$ the porous permeability parameter. With an aim to test the validity of our present analysis, we made an assumption that when $K$ and $M \longrightarrow 0$, the Eqs. (7)-(9) reduces to the classical MHD Navier-Stokes equations. The non-dimensional boundary conditions for the dimensionless velocity $u$, microrotation component $w$ and stream function $\psi(x, y)$ in the wave frame may be stated as follows:

$$
\begin{gathered}
u+\beta \frac{\partial u}{\partial y}=-1 \text { on } y=+h(x) \\
\text { and } u-\beta \frac{\partial u}{\partial y}=-1 \text { on } y=-h(x) \\
w=0 \text { on } y= \pm h(x) \\
\text { and } \psi=0 \text { on } y=0
\end{gathered}
$$

where $\beta$ is defined as the non-dimensional slip parameter and $h$ be the non-dimensional form of the upper wall whose expression is

$$
y=h(x)=1+\frac{x \tan (\theta)}{\delta}+\phi \sin (2 \pi x)
$$

with $\phi=b / a$ the amplitude ratio.

Under the assumptions of long wave length and low Reynolds number, the dimensionless equations (7)-(9) reduce to

$$
\begin{aligned}
\frac{\partial p}{\partial x} & =(1+K) \frac{\partial^{2} u}{\partial y^{2}}+K \frac{\partial w}{\partial y}-\left(H_{a}^{2}+\frac{(1+K)}{K_{p}}\right)(u+1), \\
\frac{\partial p}{\partial y} & =0, \\
M \frac{\partial^{2} w}{\partial y^{2}} & =2 K w+K \frac{\partial u}{\partial y} .
\end{aligned}
$$

Substituting the expressions for $\frac{\partial u}{\partial y}$ and $\frac{\partial^{3} u}{\partial y^{3}}$ after operating $\frac{\partial}{\partial y}$ on both sides of (12) we obtain

$$
A \frac{\partial^{4} w}{\partial y^{4}}+B \frac{\partial^{2} w}{\partial y^{2}}+C w=0
$$

The expressions for A, B and C are presented in Appendix-I.

It is observed that the Eq. (15) is a 4th order ordinary differential equation in $w$ with constant coefficients. Therefore, the general solution of $w$ takes in the form

$$
w=Z_{1} \cosh \left(\theta_{1} y\right)+Z_{2} \sinh \left(\theta_{1} y\right)+Z_{3} \cosh \left(\theta_{2} y\right)+Z_{4} \sinh \left(\theta_{2} y\right),
$$

where $\theta_{1}$ and $\theta_{2}$ together with four integrating constants $Z_{1}, Z_{2}, Z_{3}$ and $Z_{4}$ are given in Appendix-I.

Now, the expression for $u$ in terms of $w$ and its derivatives can be derived from (12) using (14) as

$$
\left\{H_{a}{ }^{2}+\frac{(1+K)}{K_{p}}\right\}(u+1)=M \frac{(1+K)}{K} \frac{\partial^{3} w}{\partial y^{3}}-(2+K) \frac{\partial w}{\partial y}-\frac{\partial p}{\partial x} .
$$




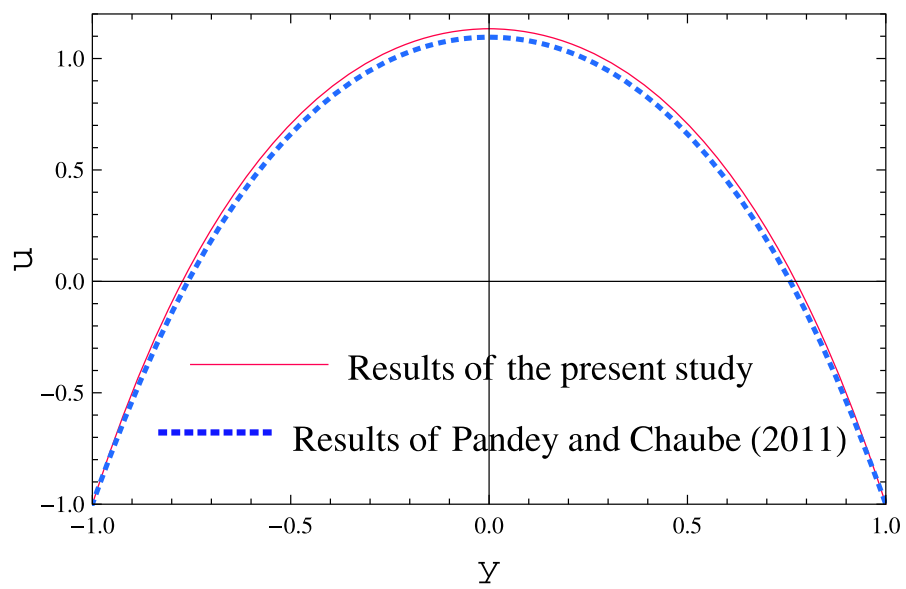

Fig. 2 Comparison of axial velocity with the results of Pandey and Chaube [3] (when $H_{a}=0.1, \phi=0.5$, $\theta=0^{\circ}, \delta=0.25, K=0.25, K_{p}=0.25, M=0.1, \beta=0.0, q=1.0$ )

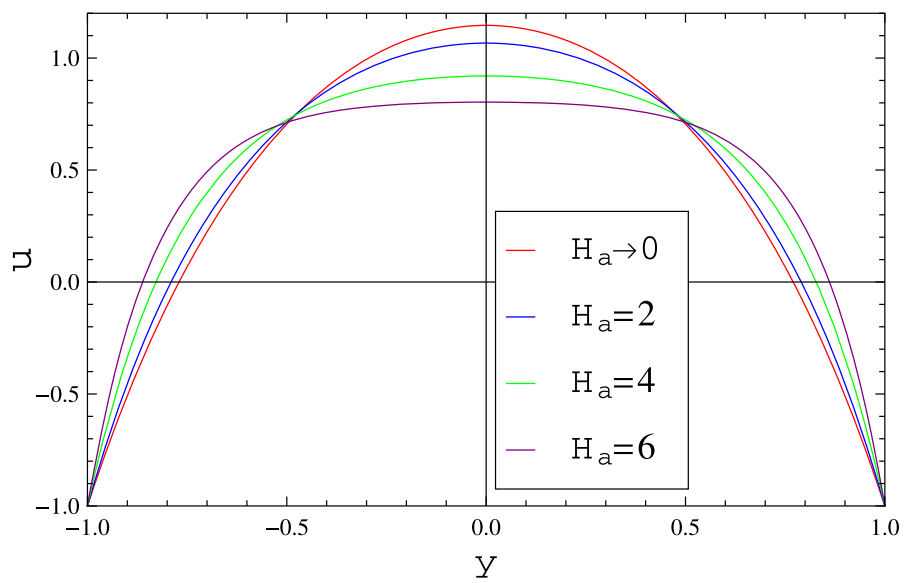

Fig. 3 Variation of axial velocity $u$ for different values of the Hartmann number $H_{a}$ with $\phi=0.5, \theta=5^{\circ}$ $\delta=0.25, K=0.2, K_{p}=0.3, M=0.3, \beta=0.0, q=1.0$

Hence the general solution for $u$ is obtained using the solution of $w$ from (17) as follows

$$
\begin{aligned}
(u+1)= & \xi_{1} Z_{1} \cosh \left(\theta_{1} y\right)+Z_{2} \xi_{1} \sinh \left(\theta_{1} y\right)+Z_{3} \xi_{2} \cosh \left(\theta_{2} y\right)+Z_{4} \xi_{2} \sinh \left(\theta_{2} y\right) \\
& -\frac{1}{\xi} \frac{\partial p}{\partial x}
\end{aligned}
$$

where the expressions for $\xi, \xi_{1}, \xi_{2}$ appeared in Eq. (18) are also given in Appendix 1.

Applying the boundary conditions given in the Eq. (10) into the Eqs. (16) and (18), the values of all the constants have been determined and are presented in the Appendix 1.

Thus, the expressions for the microrotation component $w$ and the axial component velocity $u$ are obtained as

$$
w(x, y)=\frac{\frac{\partial p}{\partial x}}{L \xi}\left[\sinh \left(\theta_{1} y\right) \sinh \left(\theta_{2} h(x)\right)-\sinh \left(\theta_{2} y\right) \sinh \left(\theta_{1} h(x)\right)\right]
$$




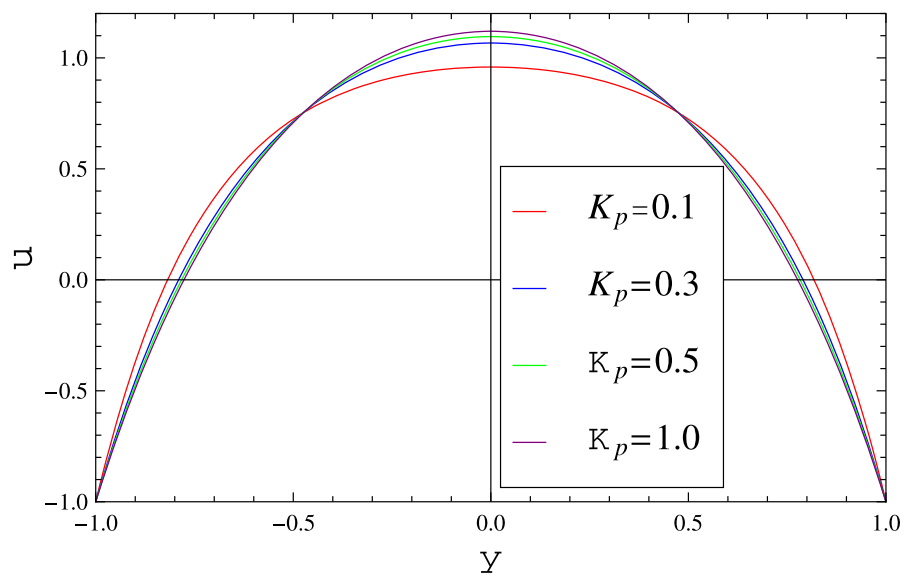

Fig. 4 Variation of axial velocity $u$ for different values of the porous permeability parameter $K_{p}$ with $\phi=0.5$, $\theta=5^{\circ} \delta=0.25, H_{a}=2, K=0.2, M=0.3, \beta=0.0, q=1.0$

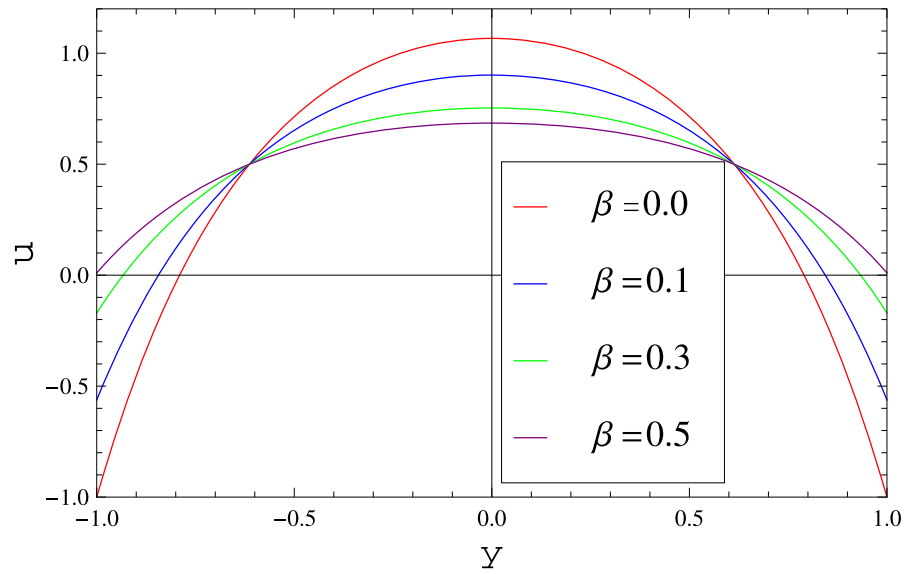

Fig. 5 Variation of axial velocity $u$ for different values of the slip parameter $\beta$ when $\phi=0.5, \theta=5^{\circ}$ $\delta=0.25, H_{a}=2, K=0.2, K_{p}=0.3, M=0.3, q=1.0$

and

$$
u(x, y)=\frac{\frac{\partial p}{\partial x}}{L \xi}\left[\xi_{1} \cosh \left(\theta_{1} y\right) \sinh \left(\theta_{2} h(x)\right)-\xi_{2} \cosh \left(\theta_{2} y\right) \sinh \left(\theta_{1} h(x)\right)-L\right]-1
$$

respectively.

Also we know that the axial velocity component $u$ in terms of stream function $\psi$ satisfy, $u=\frac{\partial \psi}{\partial y}$. Therefore, the analytic solution for streamlines can be obtained by integrating the Eq. (20) and applying boundary condition $\psi=0$ at $y=0$, as

$\psi(x, y)=\frac{\frac{\partial p}{\partial x}}{L \xi}\left[\frac{\xi_{1}}{\theta_{1}} \sinh \left(\theta_{1} y\right) \sinh \left(\theta_{2} h(x)\right)-\frac{\xi_{2}}{\theta_{2}} \sinh \left(\theta_{2} y\right) \sinh \left(\theta_{1} h(x)\right)-L y\right]-y$. 


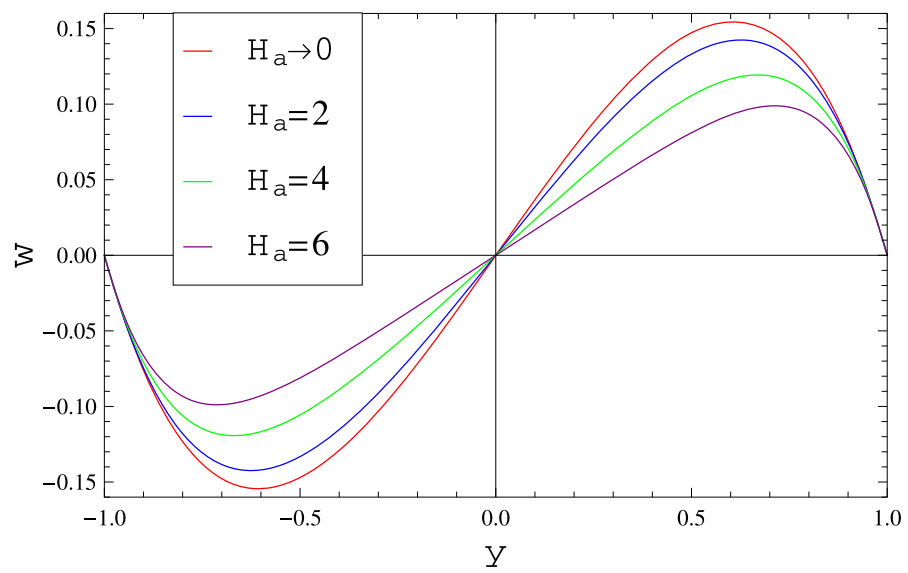

Fig. 6 Variation of microrotation component $w$ for different values of the Hartmann number $H_{a}$, with $\phi=0.5$, $\theta=5^{\circ} \delta=0.25, K=0.2, K_{p}=0.3, M=0.3, \beta=0.0, q=1.0$

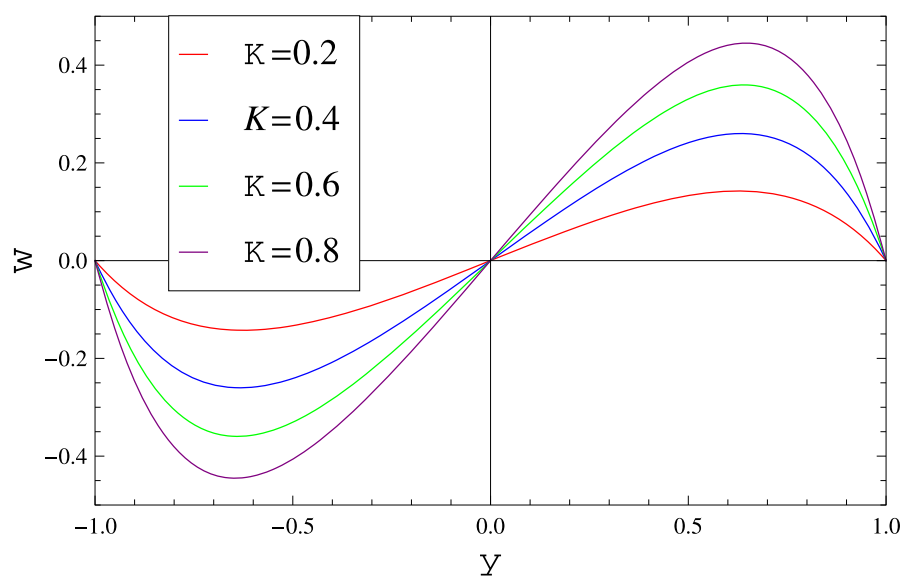

Fig. 7 Variation of microrotation component $w$ for different values of the ratio of the viscosity parameter $K$, when $\phi=0.5, \theta=5^{\circ} \delta=0.25, H_{a}=2, K_{p}=0.3, M=0.3, \beta=0.0, q=1.0$

The volumetric flow rate in the wave frame is given by

$$
q=\int_{-h(x)}^{h(x)} u(x, y) d y .
$$

In order to obtain the pumping characteristics by means of pressure rise per wavelength, the axial pressure gradient is determined from the Eq. (22) as

$$
\frac{\partial p}{\partial x}=\frac{(q+2 h(x)) L \xi}{F(x)},
$$

where the expressions for $F(x)$ is given in Appendix 1. The non-dimensional expression of pressure rise $\Delta p$ is given by,

$$
\triangle p=\int_{0}^{1} \frac{\partial p}{\partial x} d x .
$$




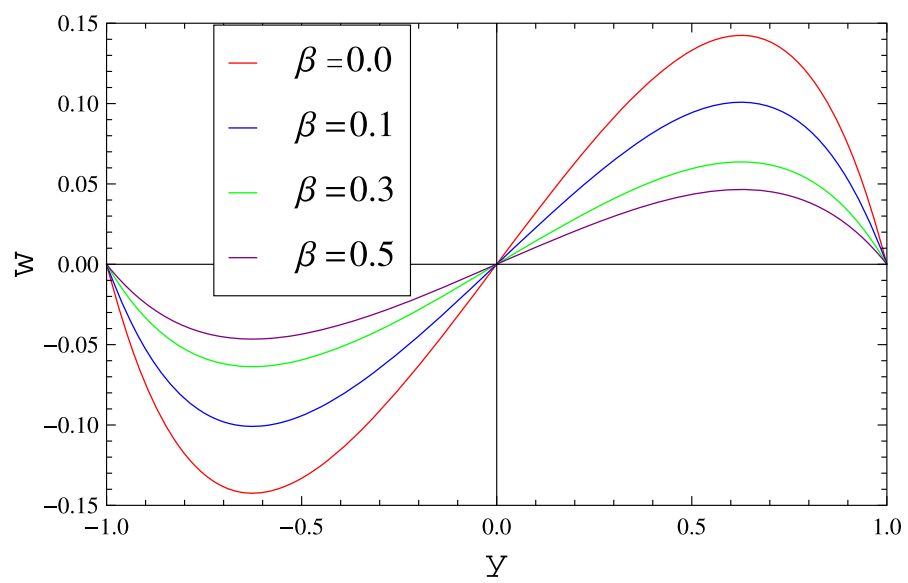

Fig. 8 Variation of microrotation component $w$ for different values of the slip parameter $\beta$, when $\phi=0.5$, $\theta=5^{\circ} \delta=0.25, H_{a}=2, K=0.2, K_{p}=0.3, M=0.3, q=1.0$

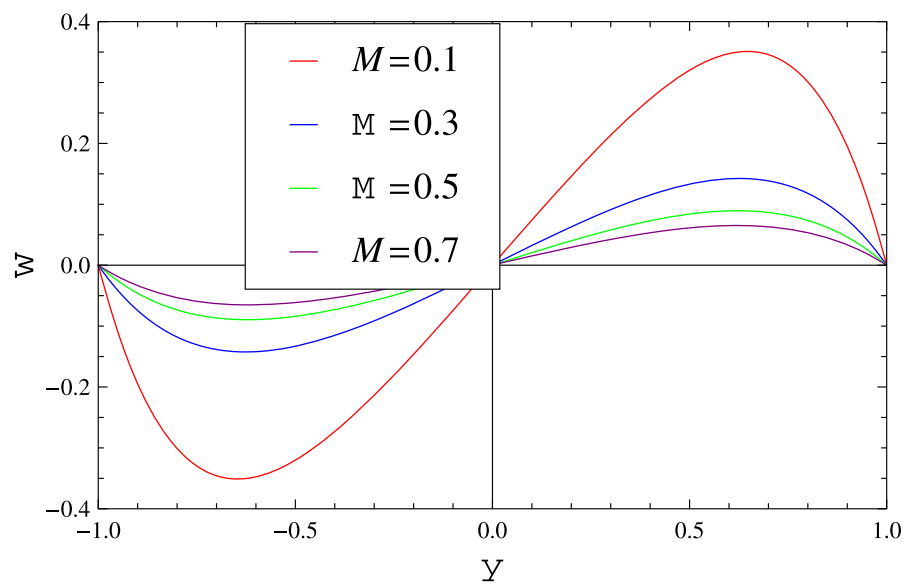

Fig. 9 Variation of microrotation component $w$ for different values of the material constant $M$, with $\phi=0.5$, $\theta=5^{\circ} \delta=0.25, H_{a}=2, K=0.2, K_{p}=0.3, \beta=0.0, q=1.0$

It is interesting to note that the stress tensor in micropolar fluid is not symmetric. Therefore, the dimensionless form of the shear stress involved in the present problem under consideration are given by

$$
\tau_{x y}=\frac{\partial u}{\partial y}-K w
$$

and

$$
\tau_{y x}=(1+K) \frac{\partial u}{\partial y}+K w
$$

The numerical computations for the shear stress $\tau_{y x}$ at the upper wall of the channel are obtained and its graphical representation is shown in the next section. 


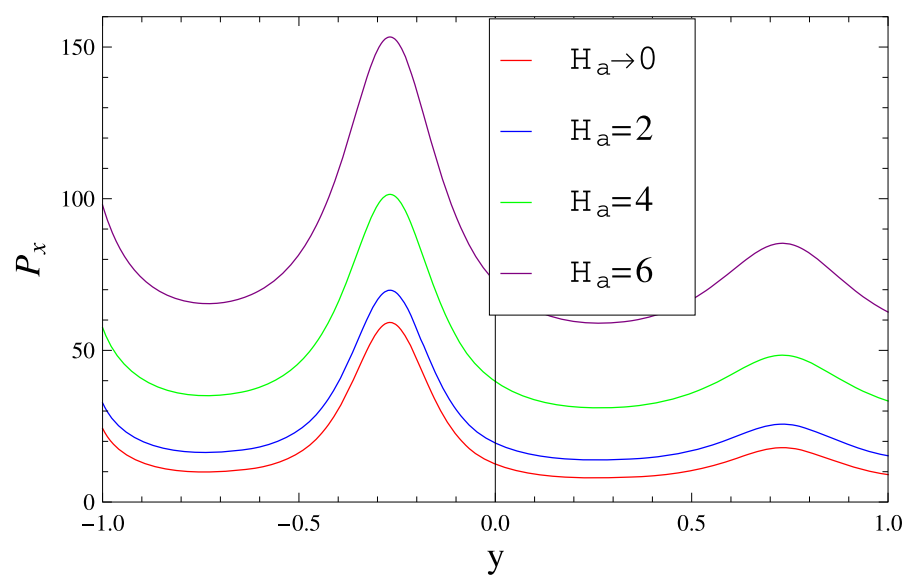

Fig. 10 Distribution of pressure gradient $P_{x}=\frac{\partial P}{\partial x}$ for different values of the Hartmann number $H_{a}$ with $\phi=0.5, \theta=5^{\circ} \delta=0.25, K=0.2, K_{p}=0.3, \stackrel{\partial}{M}=0.3, \beta=0.0, q=1.0$

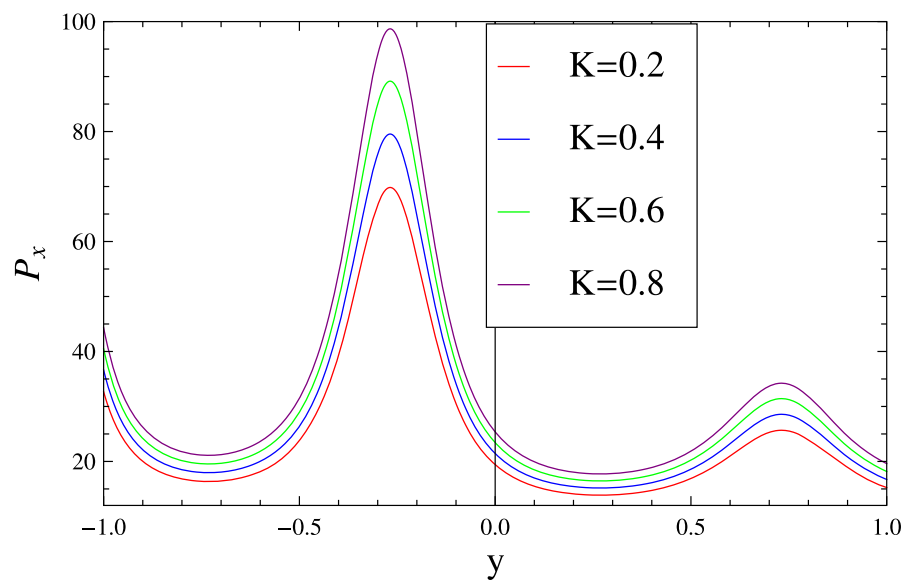

Fig. 11 Distribution of pressure gradient $P_{x}=\frac{\partial P}{\partial x}$ for different values of $K$ with $\phi=0.5, \theta=5^{\circ}, \delta=0.25$, $H_{a}=2, K_{p}=0.3, M=0.3, \beta=0.0, q=1.0$

\section{Computational Results and Discussion}

In the previous section, we have obtained analytical expressions for the axial velocity components, microrotation components, pressure gradient, volumetric flow rate and stream function for the problem under consideration. In this section, the computational results have been obtained and presented them graphically using the following set of values as recommended by $([5,13,19,23,29,39])$ :

$$
\begin{aligned}
& \phi=0.5, \theta=0^{\circ}, 5^{\circ}, 10^{\circ}, 15^{\circ} ; \delta=0.25 ; H_{a}=0,2,4,6 ;, K=0.2,0.4,0.6,0.8 ; \\
& K_{p}=0.1,0.3,0.5,1.0 ; M=0.1,0.3,0.5,0.7 ; \beta=0.0,0.1,0.3,0.5 ; q=1.0 .
\end{aligned}
$$

With an aim to validate our numerical results, the axial velocity $u$ presented in Fig. 2 is compared with the results of Pandey and Chaube [29] by considering same flow configuration. It shows that our results are in good agreement. Figures 3, 4, 5 represent the variation of 


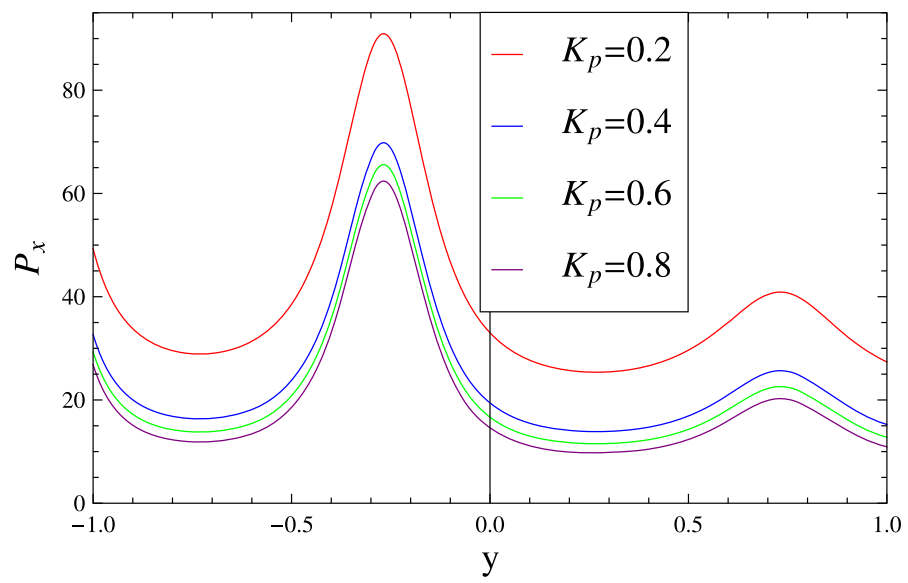

Fig. 12 Distribution of pressure gradient $P_{x}=\frac{\partial P}{\partial x}$ for different values of $K_{p}$ with $\phi=0.5, \theta=5^{\circ} \delta=0.25$, $H_{a}=2, K=0.2, K_{p}=0.3, M=0.3, \beta=0.0, q=1.0$

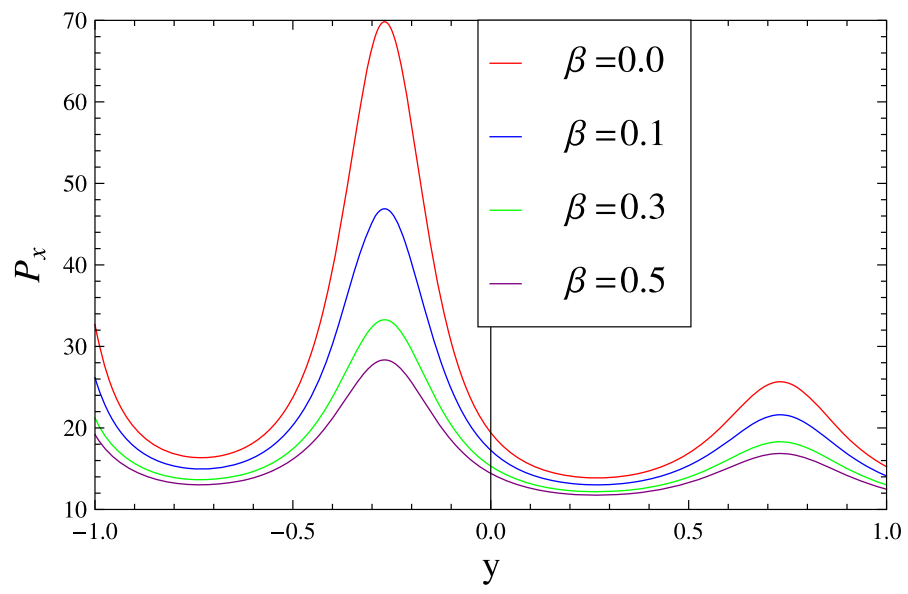

Fig. 13 Distribution of pressure gradient $P_{x}=\frac{\partial P}{\partial x}$ for different values of $\beta$ with $\phi=0.5, \theta=5^{\circ} \delta=0.25$, $H_{a}=2, K=0.2, K_{p}=0.3, M=0.3, q=1.0$

axial velocity across the height of the channel at $x=0$ for different values of the physical parameters of interest. Figure 3 shows that the axial velocity decreases and more flattening is observed at the central line of the channel with the increase of the Hartmann number $\left(H_{a}\right)$, whereas the axial velocity has an enhancing effect near the channel walls in order to maintain constant flow rate. Due to the application of an external magnetic field in the direction of perpendicular to the axial flow, inducing electric currents inside the flow domain and thereby arises Lorentz force. This force has a tendency to slow down the motion of the fluid. In Fig. 4 we observe that the axial velocity also decreases at the central line of the channel for the increasing of porous permeability parameter $K_{p}$, while the opposite trend is observed in the vicinity of the channel walls. The variation of the axial velocity $u$ for different values of slip parameter $\beta$ have presented in Fig. 5. It is interesting to note from this figure that the axial velocity at the central line of the channel also decreases with increasing values of slip 


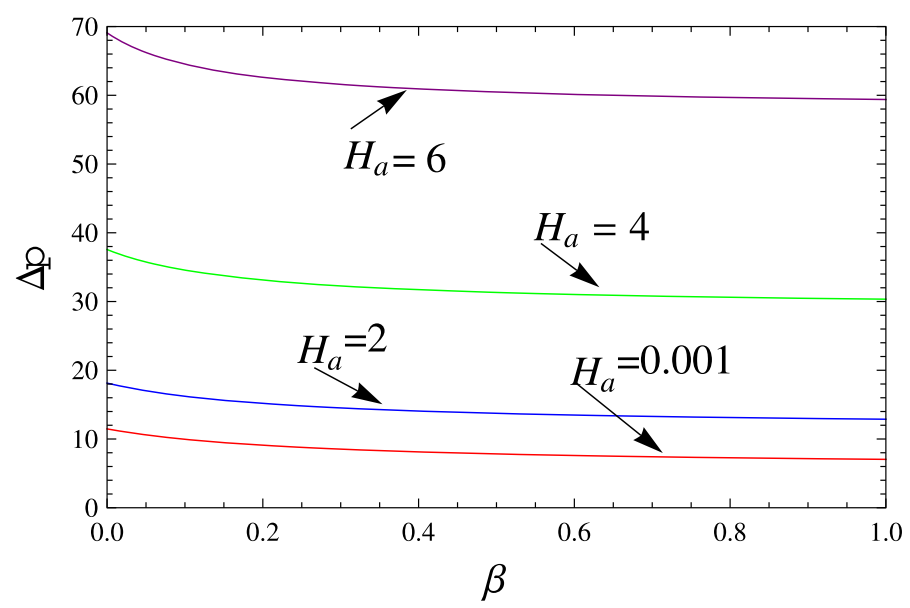

Fig. 14 Variation of pressure rise $\Delta P$ with slip parameter $\beta$ for different values of $H_{a}$ with $\phi=0.5, \theta=5^{\circ}$ $\delta=0.25, H_{a}=2, K=0.2, K_{p}=0.3, M=0.3, \beta=0.0, q=1.0$

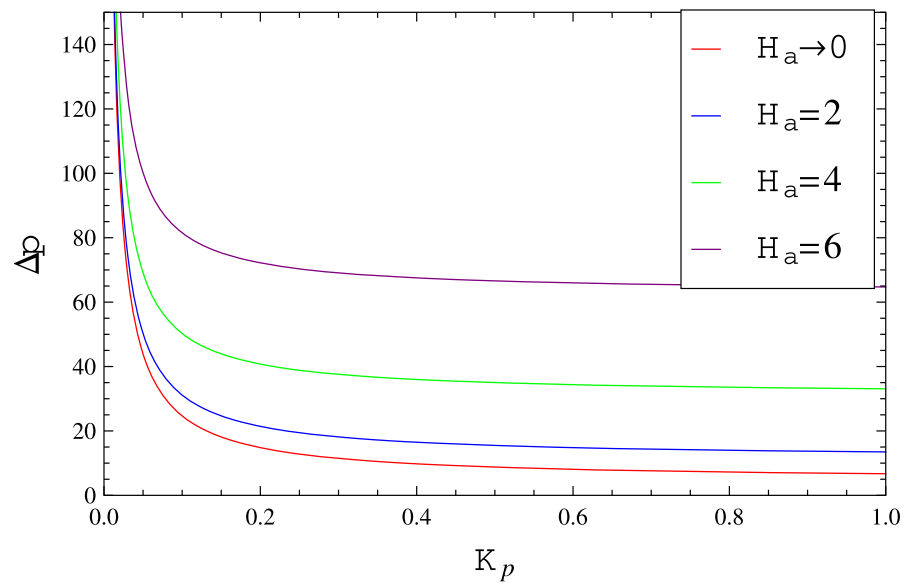

Fig. 15 Variation of pressure rise $\Delta P$ with porous permeability parameter $K_{p}$ for different values of Hartmann number $H_{a}$ with $\phi=0.5, \theta=5^{\circ} \delta=0.25, K=0.2, \beta=0.0, M=0.3, q=1.0$

parameter $\beta$. However, the axial velocity in the vicinity of the channel walls is dissimilar because of the presence of slip parameter $\beta$. It is also important to note from this figure that due to the presence of slip parameter $\beta$, the flow reversal eliminated at the channel walls.

Another important kinematic characteristic of micropolar fluid is the microrotation component $w$ of microparticles suspended in the fluid itself. The distributions of microrotation component are presented in Figs. 6, 7, 8, 9 for different values of the non-dimensional parameters. Figure 6 deals with the variation of Hartmann number $H_{a}$ on the microrotation component $w$. We observe that the magnitude of the microrotation component $w$ decreases on one side of the channel with the increase of the Hartmann number $H_{a}$, while on the other side of the channel, $w$ increases with the increase of $H_{a}$. This indicates that the microparticles rotates in two different directions about the central line of the channel and the center of rotation shifted to the wall with increasing magnetic field strength i.e., Hartmann number 


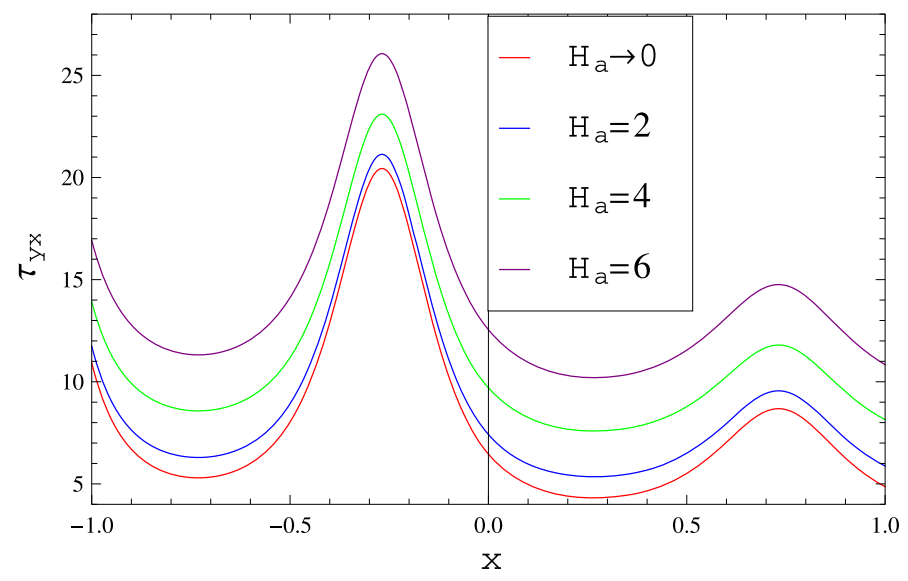

Fig. 16 The variation of wall shear stress $\tau_{y x}$ at the upper wall of the channel for different values of $H_{a}$, with $\phi=0.5, \theta=5^{\circ} \delta=0.25, K=0.2, K_{p}=0.3, M=0.3, \beta=0.0, q=1.0$

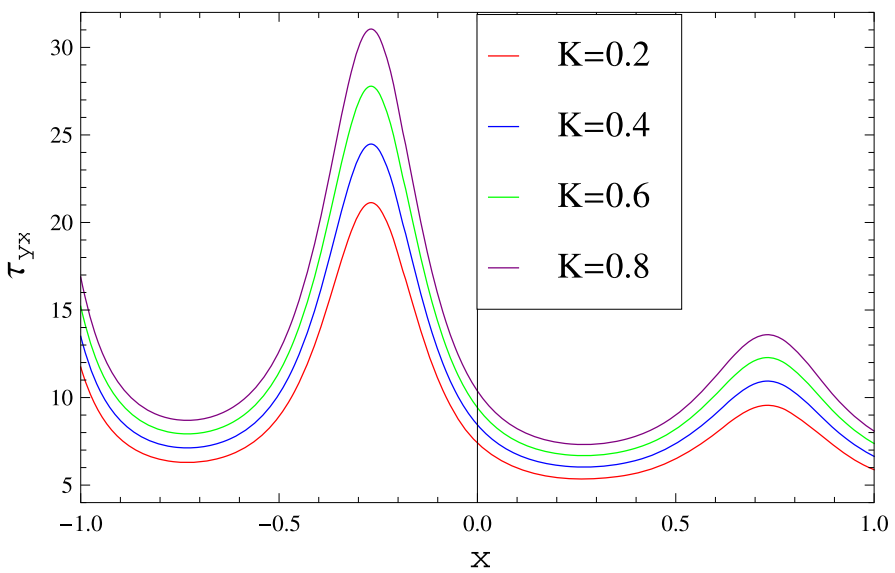

Fig. 17 The variation of wall shear stress $\tau_{y x}$ at the upper wall of the channel for different values of $K$, when $\phi=0.5, \theta=5^{\circ} \delta=0.25, H_{a}=2, K_{p}=0.3, M=0.3, \beta=0.0, q=1.0$

$H_{a}$. Figure 7 reveals that the magnitude of the microrotation component increases on the upper side of the channel with the increasing values of the ratio of viscosity parameter $K$, whereas the opposite trend is observed on the lower portion of the channel. However, the variation of microrotation component on the slip parameter $\beta$ and micropolar parameter (known as particle size parameter ) $M$ are identical as shown in Figs. 8 and 9. When the particle size increases i.e., micropolar parameter increases, the microrotation of the microcomponent decreases. Moreover, the microrotation occurs maximum in the case of no-slip condition at the wall. One can observe here that the higher values of slip parameter causes unable to rotate microparticles in the vicinity of the channel walls.

Figures 10, 11, 12, 13 illustrate the variation of axial pressure gradient $\frac{\partial p}{\partial x}$ in two wave length for different values of the Hartmann number $H_{a}$, the ratio of viscosity parameter $K$, the porous permeability parameter $K_{p}$ and the slip parameter $\beta$. Figure 10 reveals that the axial pressure gradient increases with increasing magnetic field strength i.e., increasing of 


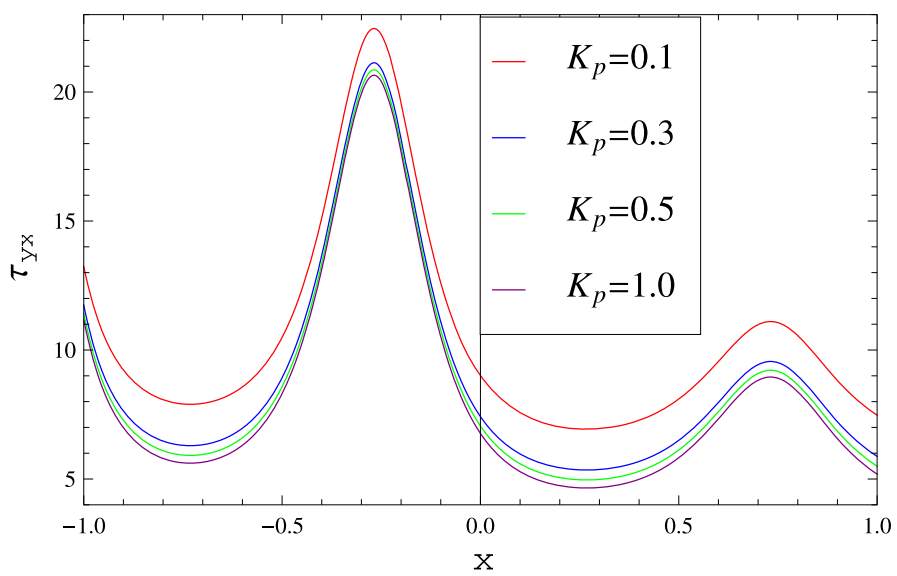

Fig. 18 The variation of wall shear stress $\tau_{y x}$ at the upper wall of the channel for different values of $K_{p}$ With $\phi=0.5, \theta=5^{\circ} \delta=0.25, H_{a}=2, K=0.2, M=0.3, \beta=0.0, q=1.0$

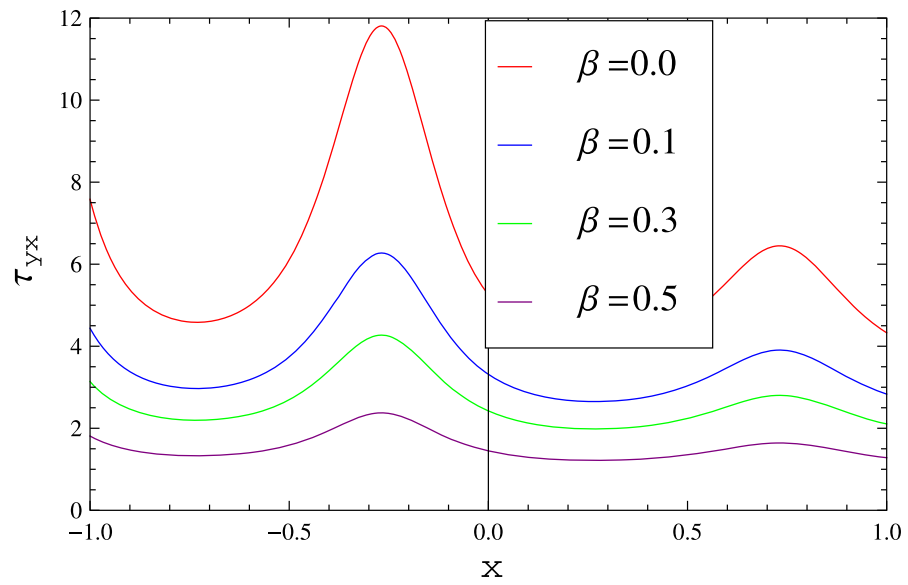

Fig. 19 The variation of wall shear stress $\tau_{y x}$ at the upper wall of the channel for different values of $\beta$ with $\phi=0.5, \theta=5^{\circ} \delta=0.25, H_{a}=2, K=0.2, K_{p}=0.3,, M=0.3, q=1.0$

Hartmann number $H_{a}$. As the velocity decreases thereby pressure gradient increases in the presence of magnetic field in order to maintain constant flow rate. It has been shown in Fig. 11 that the axial pressure gradient increases with the increase of the ratio of viscosity parameter $K$. The increase of classical viscosity leads to the increase of the ratio of viscosity parameter $K$ and thereby increases the pressure gradient. From Figs. 12 and 13 we observe that the axial pressure gradient decreases with the increase of the porous permeability parameter $K_{p}$ as well as the slip parameter $\beta$. Therefore the slip effects has a tendency to reducing the pressure gradient. One can note from all these figures that the magnitude of the peak value of the pressure gradient decreases along the length of the distensible tube. It is also observed from these figures that in the wider part of the channel the pressure gradient is relatively small, where the flow can easily pass without giving any large pressure gradient. While the large pressure gradient is required to maintain the same flow rate to pass it in the narrow part of the channel. The variation of pressure rise $\triangle p$ with slip parameter $\beta$ and porous permeability 

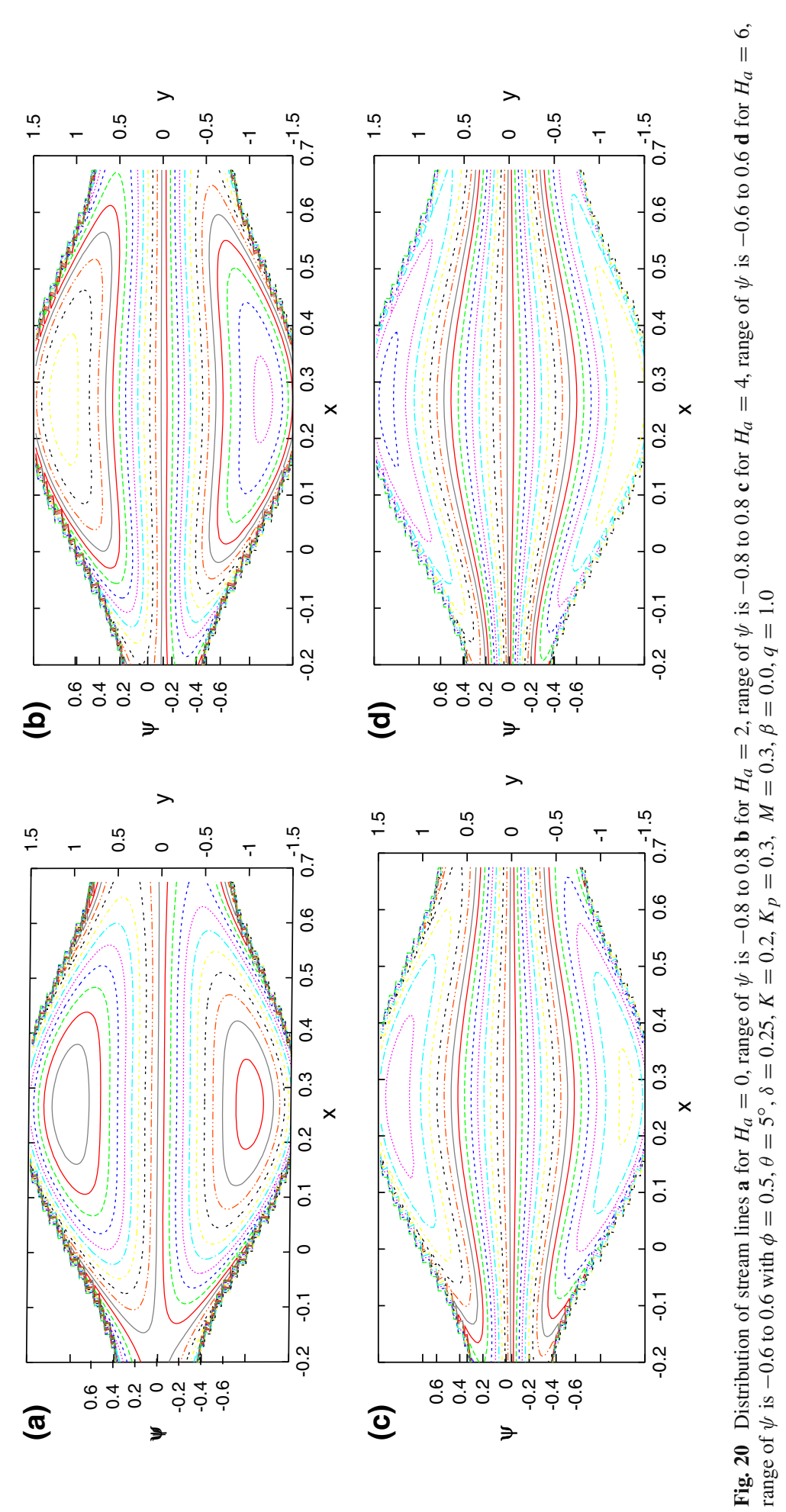

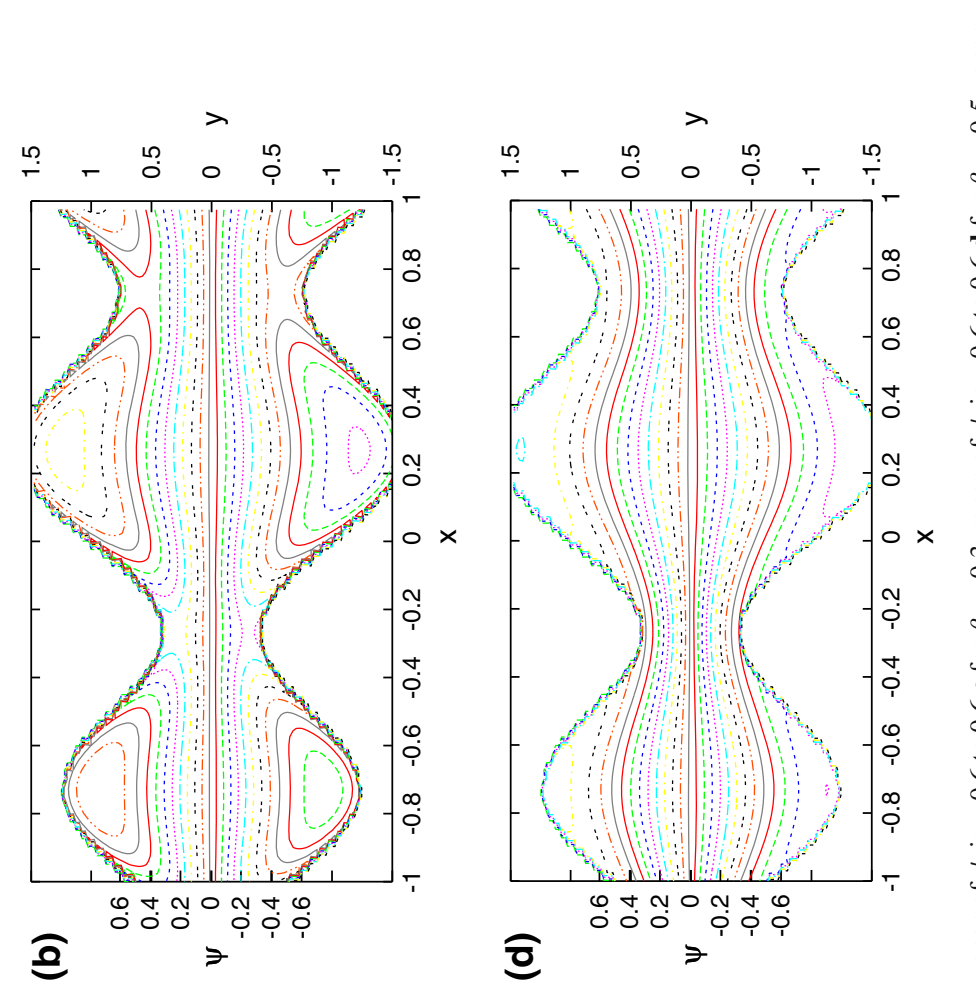

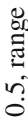
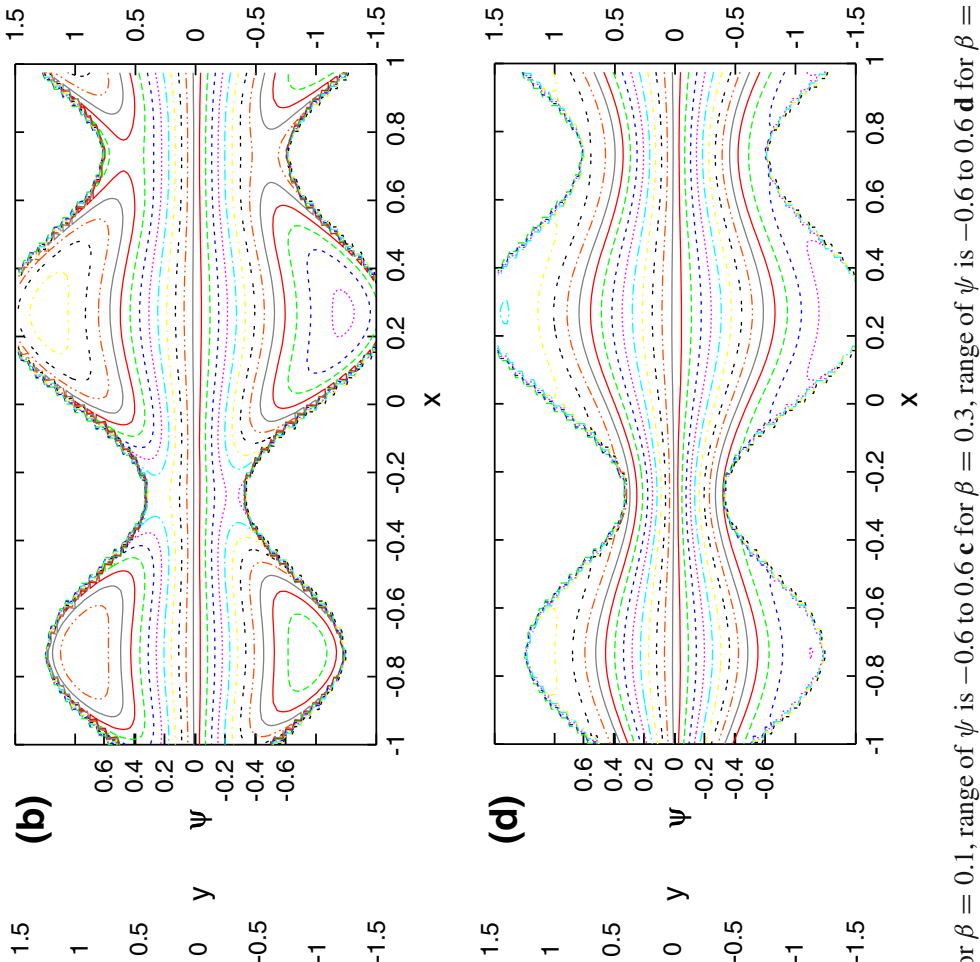

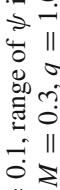

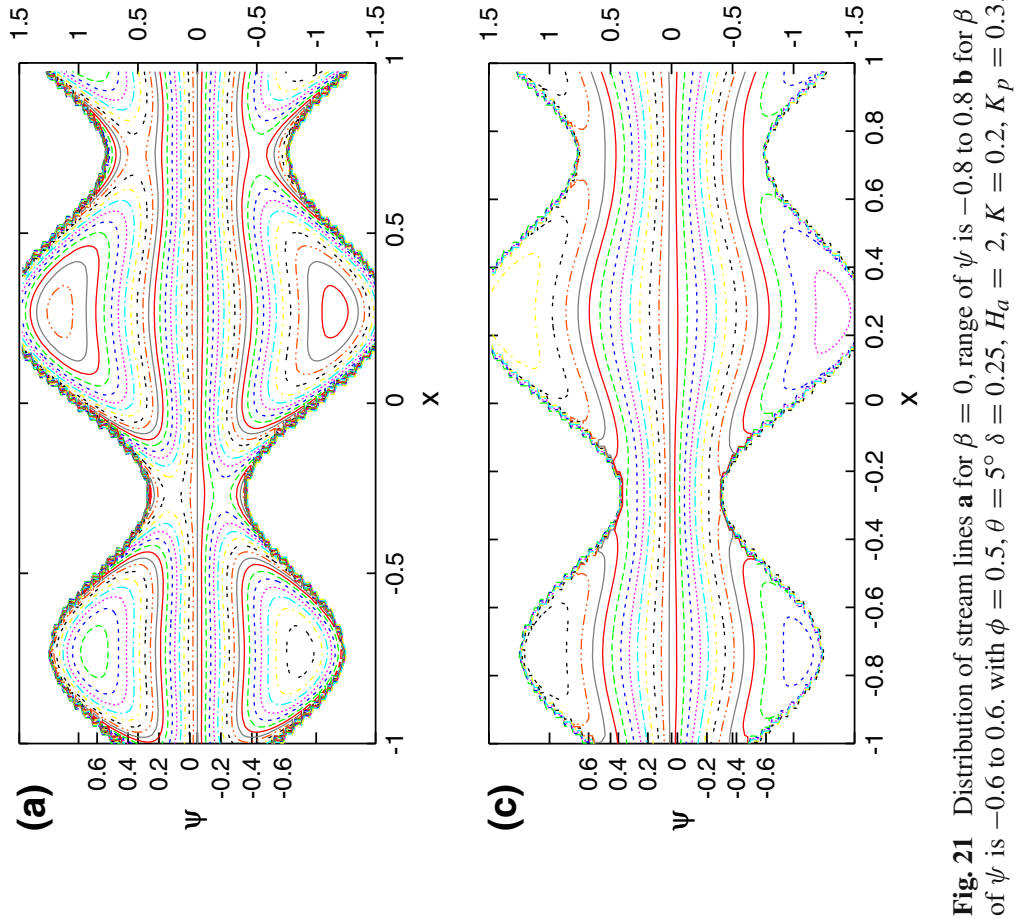




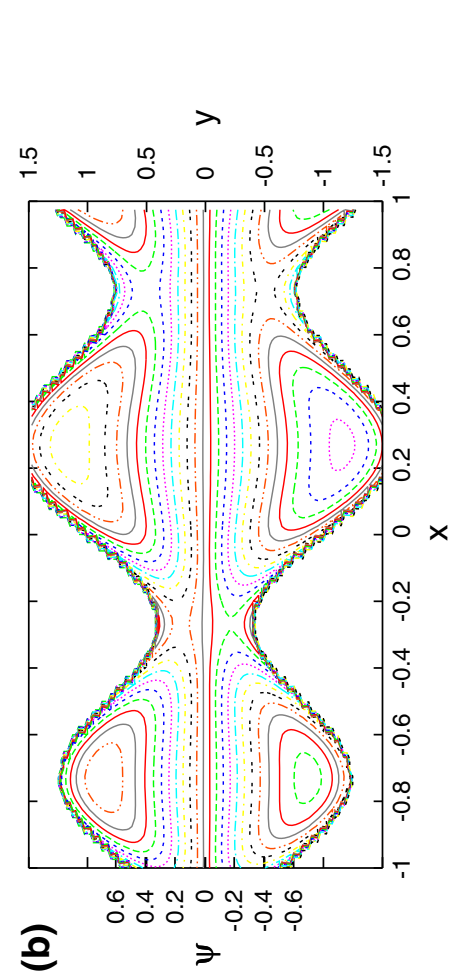

है

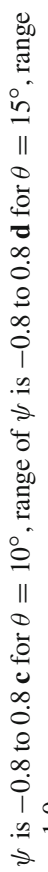
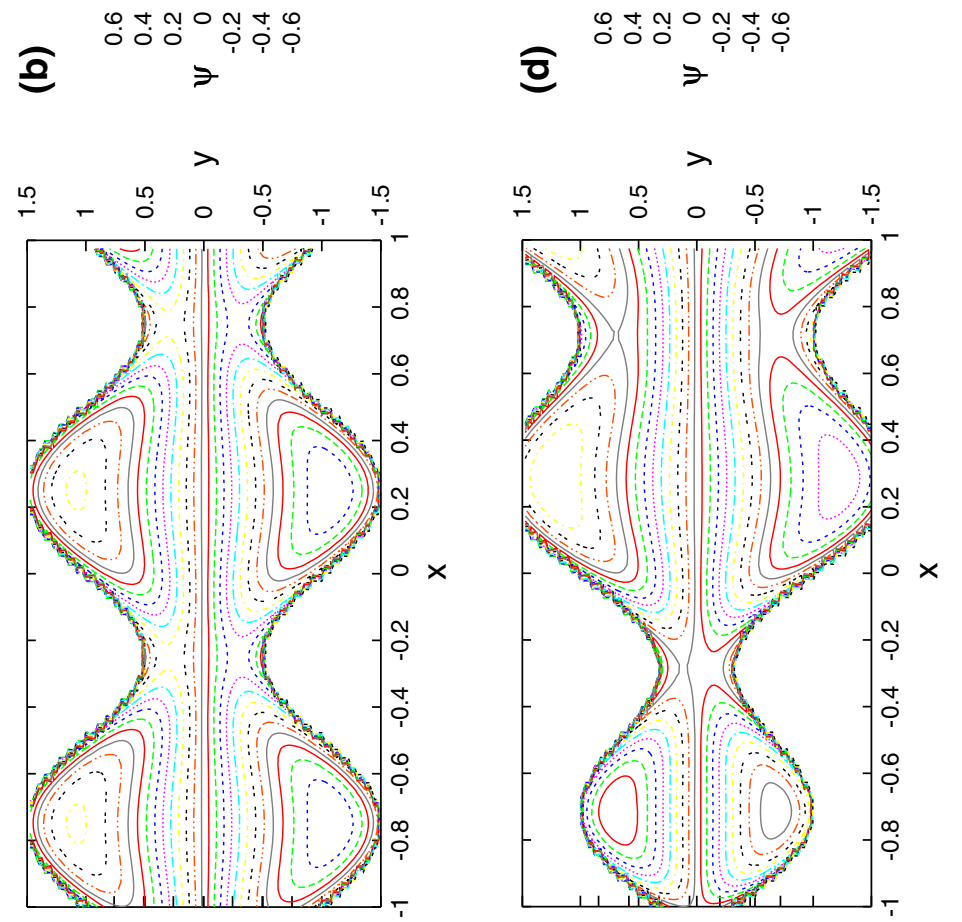

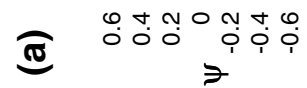

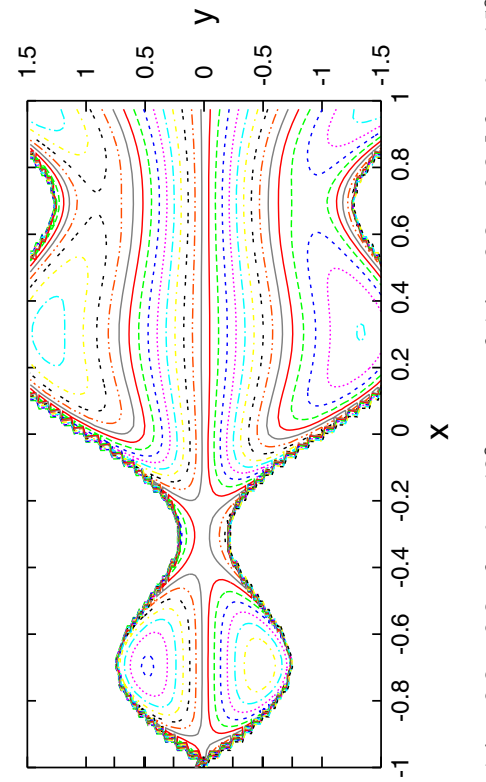

$\stackrel{0}{11}$

पै

$\infty$

है II

in $x$

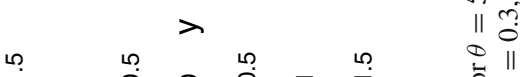

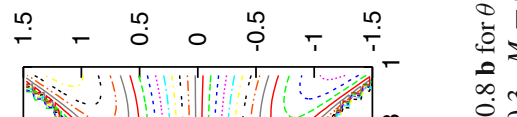

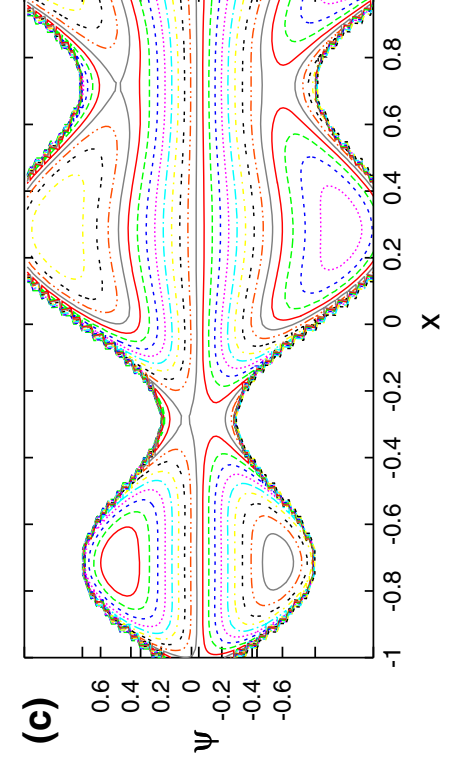

$\begin{array}{cc}\infty & m \\ 0 & 0 \\ 0 & 11 \\ \infty & 1\end{array}$

$\infty_{1}^{\infty} \leqslant$

$\therefore$ ก

$\gg 0$

to

$\infty_{\infty}^{\infty} \times$

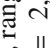

$\therefore$

11

จิ กิ

인

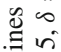

छ

Ð

恋证

है

$\stackrel{0}{\circ}$

을

告文

สี

我艺 


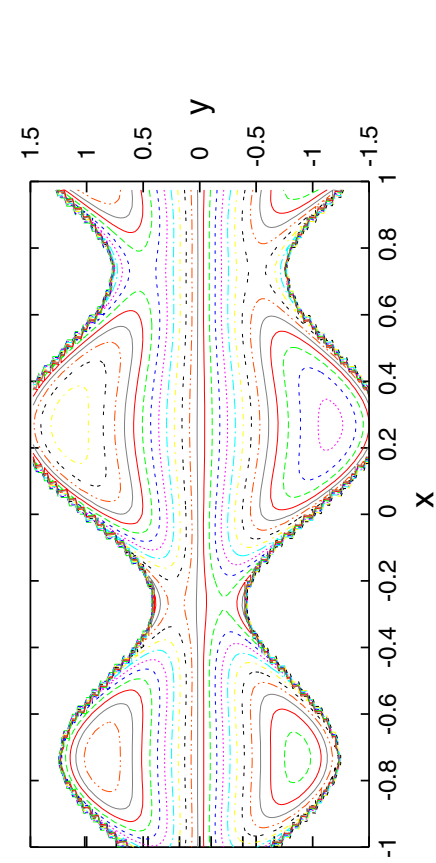

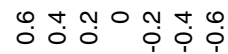

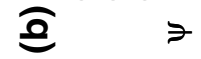

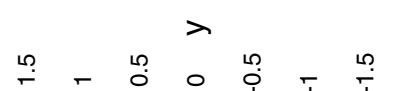
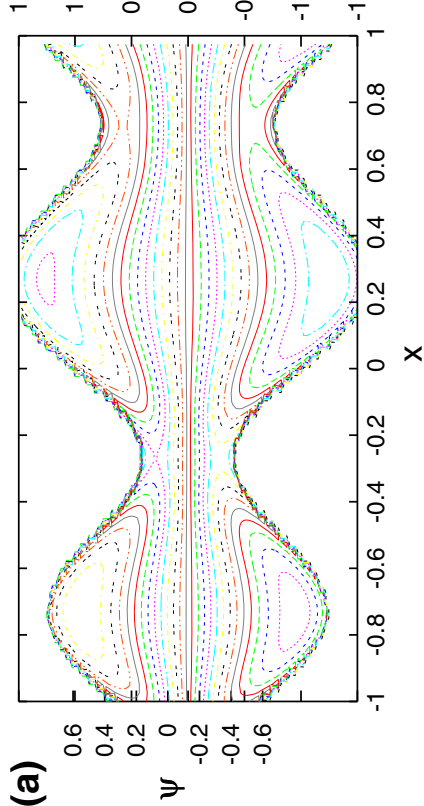

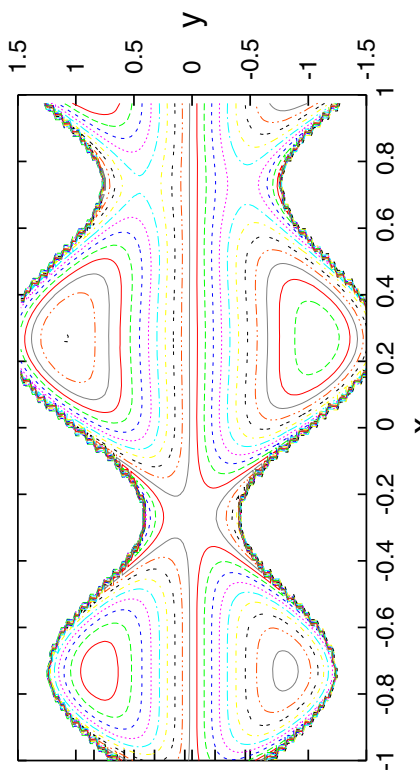

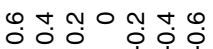
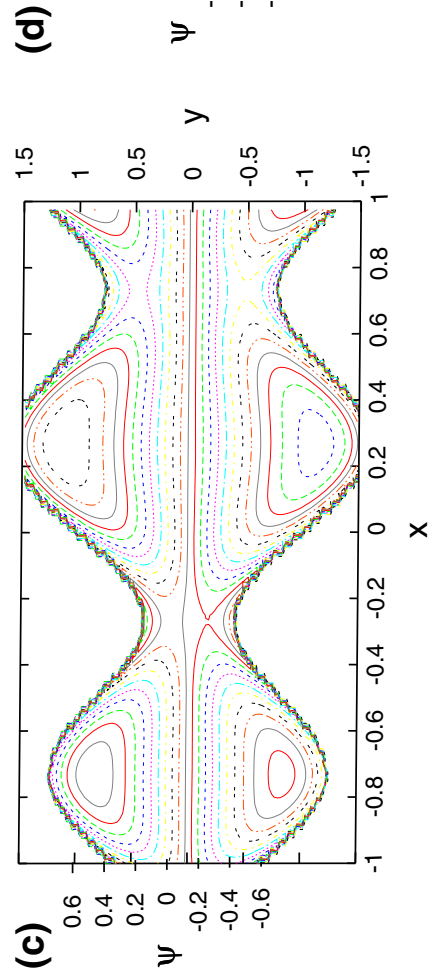

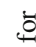

๘

$\stackrel{\infty}{0}$

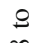

$\stackrel{\infty}{i}$

$\mathscr{2}$

$+$

(1)

ถ્อ

?

II

v

อี

$\infty$

0

$\infty$

@

$\approx$

$\rightarrow 11$

40

80

踏

लं

11

ख̃

ㅎํ ก

- 11

$\stackrel{0}{0}$

을

@

$1 \geqslant$

$\rightarrow 2$

पे

00

矛 in

$=11$

00

11 r

₹

흥

的

¿

$\Xi 0$

ฐ

む્

4.2

흥

范

龺。

त I

象远 
parameter $K_{p}$ for different strength of the magnetic field are shown in Figs. 14 and 15. In both the cases pressure rise increases with the increase of the Hartmann number $H_{a}$. The pressure rise decreases asymptotically with the increase of the slip parameter $\beta$ as well as the porous permeability parameter $K_{p}$. It is also interesting to note that the pressure rise changes rapidly within a small range of both $\beta$ and $K_{p}$ beyond which no change is observed.

The variation of wall shear stress $\tau_{y x}$ at the upper wall of the channel for different values of the physical parameters is depicted in Figs. 16, 17, 18, 19. It has been observed from Figs. 16 and 17 that the wall shear stress increases with the increase of the Hartmann number $H_{a}$ and the ratio of viscosity parameter $K$. The small changes in $K$ leads to large changes in the wall shear stress than that of magnetic field strength. Therefore, microrotation viscosity play a vital role on the wall shear stress. Figure 18 shows that the wall shear stress decreases with the increase of the porous permeability parameter $K_{p}$. It is interesting to note from Fig. 19 that the wall shear stress significantly decreases in the presence of slip parameter $\beta$. It reveals that in the absence of slip effect, the wall shear stress increases at the narrowing part of the channel, while the wall shear stress is minimum at the wider part of the channel. However, in the presence of large slip parameter $\beta$, the wall shear stress becomes almost linear.

Owing to the high flow rates and large occlusions there forms a region of closed streamlines in the wave frame known as bolus. The bolus was found to move with the wave speed and there occurs stagnation points where both the velocity components vanish in the wave frame. The distribution of stream lines pattern in the presence of magnetic field are shown in Fig. 20 in one wave length. We observe that as the Hartmann number $H_{a}$ increases the formation of bolus at the wall decreases in size. It is interesting to note from Fig. 21 that the trapped bolus decreases in size and ultimately vanishes for increasing values of slip parameter $\beta$. Thus, the magnetic field strength and the slip effects helps to restrict the formation of the trapped bolus. Figure 22 gives the distribution of streamlines for different diverging angle $\theta$. We observe that as $\theta$ increases the trapped bolus found to increase in size on both sides of the central line of the channel. However, the porous permeability parameter $K_{p}$ keeps to form more closed streamlines at the wall as shown in Fig. 23.

\section{Conclusions}

In this paper, an attempt has been made to investigate the effects of slip velocity on the peristaltic transport of physiological fluids represented by a micropolar fluid model passing through a non-uniform porous channel. In this investigation, special emphasis has been paid to the study of velocity distribution, the pumping characteristics and the trapping phenomena. From the present analysis, one can make an important conclusion that it is possible to increase pumping action (pressure gradient) as often as necessary by applying an external magnetic field and that the bolus formation can be eliminated with a considerable extent. The wall shear stress $\tau_{y x}$ increases with the increase of Hartmann number $H_{a}$ at the upper wall. The slip velocity at the wall has reducing effect on the formation of trapped bolus. Thus the results presented here throws some light on problems associated with fluid movement in the gastrointestinal tract, intra-uterine fluid motion induced by uterine contraction, as well as flow through small blood vessels and intrapleural membranes.

Acknowledgments The authors are grateful to the esteemed reviewers for their suggestions based on which the present manuscript is improved. One of the authors (G. C. Shit) is thankful to SERB, Department of 
Science and Technology (DST), Govt. of India for the financial support through project research grant during this investigation.

\section{Appendix 1}

The expressions that appear in "Mathematical Formulation" section are listed as follows:

$$
\begin{aligned}
A= & M(1+K), B=-\left[\left(2 K+K^{2}\right)+M\left\{H_{a}^{2}+\frac{(1+K)}{K_{p}}\right\}\right] \\
C= & 2 K\left\{H_{a}^{2}+\frac{(1+K)}{K_{p}}\right\}, \\
\theta_{1}= & \frac{1}{\sqrt{2 A}} \sqrt{-B+\sqrt{B^{2}-4 A C}}, \quad \theta_{2}=\frac{1}{\sqrt{2 A}} \sqrt{-B-\sqrt{B^{2}-4 A C}}, \\
\xi= & \left\{H_{a}^{2}+\frac{(1+K)}{K_{p}}\right\} \quad \xi_{1}=\frac{M(1+K)}{K \xi} \theta_{1}^{3}-\frac{(2+K)}{\xi} \theta_{1}, \\
& \xi_{2}=\frac{M(1+K)}{K \xi} \theta_{2}^{3}-\frac{(2+K)}{\xi} \theta_{2}, \\
L= & \left(\xi_{1} \cos h\left(\theta_{1} h(x)\right)+\beta \xi_{1} \theta_{1} \sin h\left(\theta_{1} h(x)\right)\right) \sin h\left(\theta_{2} h(x)\right)-\left(\xi_{2} \cos h\left(\theta_{2} h(x)\right)\right. \\
& \left.+\beta \xi_{2} \theta_{2} \sin h\left(\theta_{2} h(x)\right)\right) \sin h\left(\theta_{1} h(x)\right) \\
Z_{1}= & 0=Z_{3}, \quad Z_{2}=\frac{1}{L \xi} \frac{\partial p}{\partial x} \sin h\left(\theta_{2} h(x)\right), \quad Z_{4}=-\frac{1}{L \xi} \frac{\partial p}{\partial x} \sin h\left(\theta_{1} h(x)\right), \\
F(x)= & 2\left(\frac{\xi_{1}}{\theta_{1}}-\frac{\xi_{2}}{\theta_{2}}\right) \sin h\left(\theta_{1} h(x)\right) \sin h\left(\theta_{2} h(x)\right)-2 L h(x)
\end{aligned}
$$

\section{References}

1. Ali, N., Hayat, T.: Peristaltic flow of a micropolar fluid in an asymmetric channel. Comput. Math. Appl. 55, 589-608 (2008)

2. Ariman, T., Turk, M.A., Sylvaster, N.D.: Review article: applications of microcontinuum fluid mechanics. Int. J. Eng. Sci. 12, 273-293 (1974)

3. Chaube, M.K., Pandey, S.K., Tripathi, D.: Slip effect on peristaltic transport of micropolar fluid. Appl. Math. Sci. 4, 2105-2117 (2010)

4. Ellahi, R.: Effects of the slip boundary condition on non-Newtonian flows in a channel. Commun. Nonlinear Sci. Numer. Simul. 14(4), 1377-1384 (2009)

5. Ellahi, R., Hayat, T., Mahomed, F.M., Asghar, S.: Effects of slip on the non-linear flows of a third grade fluid. Nonlinear Anal. Ser. B 11, 139-146 (2010)

6. Ellahi, R., Riaz, A., Naddem, S., Ali, M.: Peristalic flow of Carreau fluid in a rectangular duct through a porous medium. Math. Probl. Eng. 2012, 1-24 (2012). Article ID 329639

7. El-Shehawey, E.F., Husseny, S.Z.A.: Effect of porous boundaries on Peristaltic transport through porous medium. Acta Mech. 143, 165-177 (2000)

8. Eringen, A.C.: Theory of micropolar fluids. J. Math. Mech. 16, 1-18 (1966)

9. Eytan, O., Elad, D.: Analysis of intra-Urterine fluid motion induced by uterine contractions. Bull. Math. Biol. 61, 221-238 (1999)

10. Hayat, T., Ali, N., Asghar, S.: Hall effect on Peristaltic flow of a Maxwell fluid in a porous medium. Phys. Lett. A 363, 397-403 (2007)

11. Hayat, T., Qureshi, M.U., Ali, N.: The influence of slip on peristaltic motion of a third order fluid in an asymmetric channel. Phys. Lett. A 372, 2653-2664 (2008) 
12. Hung, T.K., Brown, T.D.: Solid-particle motion in two dimensional peristaltic flows. J. Fluid Mech. 73, 77-96 (1976)

13. Jafrin, M.Y., Shapiro, A.H.: Peristaltic pumping. Annu. Rev. Fluid Mech. 3, 13-37 (1971)

14. Khan, A.A., Ellahi, R., Vafai, K., Peristaltic transport of Jeffrey fluid with variable viscosity through a porous medium in an asymmetric channel. Adv. Math. Phys. (2012), 1-15, Article ID 169642, (2012). doi: $10.1155 / 2012 / 169642$

15. Kothandapani, M., Srinivas, S.: Non-linear peristaltic transport of Newtonian fluid in an inclined assymmetric channel through porous medium. Phys. Lett. A 372, 1265-1276 (2008)

16. Latham, T.W.: Fluid motion in a peristaltic pumps, M. S. Thesis, MIT, Cambridge (1966)

17. Lee, J.S., Fung, Y.C.: Flow in a non-uniform small blood vessels. Microvasc. Res. 3, 272-287 (1971)

18. Lukaszewicz, G.: Micropolar Fluids: Theory and Applications. Birkhauser, New York (1999)

19. Maiti, S., Misra, J.C.: Peristaltic flow of a fluid in a porous channel: a study having relevance to flow of bile within ducts in a pathological state. Int. J. Eng. Sci. 49(9), 50-966 (2011)

20. Mathu, P., Rathiskumar, B.V., Chandra, P.: Peristaltic motion of micropolar fluid in circular cylindrical tubes: effect of wall properties. Appl. Math. Model. 32, 2019-2033 (2008)

21. Mekheimer, KhS, El Shehawey, E.F., Elaw, A.M.: Peristaltic motion of a particle-fluid suspension in a planar channel. Int. J. Theor. Phys. 37, 2895-2920 (1998)

22. Mekheimer, KhS, Al-Arabi, T.H.: Non-linear peristaltic transport of MHD flow through a porous medium. Int. J. Math. Math. Sci. 26, 1663-1682 (2003)

23. Mishra, M., Rao, A.R.: Peristaltic transport of a Newtonian fluid in an asymmetric channel. Zeitschrift fr angewandte Mathematik und Physik (ZAMP) 54, 532-550 (2003)

24. Rao, A.R., Mishra, M.: Non-linear and curvature effects on peristaltic flow of a viscous fluid in an asymmetric channel. Acta Mech. 168, 35-59 (2004)

25. Misra, J.C., Pandey, S.K.: Peristaltic transport of a particle-fluid suspension in a cylindrical tube. Comput. Math. Appl. 28, 131-145 (1994)

26. Misra, J.C., Pandey, S.K.: Peristaltic transport of blood in small vessels: study of mathematical model. Comput. Math. Appl. 43, 1183-1193 (2002)

27. Misra, J.C., Maiti, S., Shit, G.C.: Peristaltic transport of a physiological fluid in an asymmetric porous channel in the presence of an external magfnetic field. J. Mech. Med. Biol. 8, 507-525 (2008)

28. Nadeem, S., Riaz, A., Ellahi, R., Akbar, N.S.: Series solution of unsteady peristaltic flow of a Carreau fluid in eccentric cylinders. Ain Shams Eng. J. 5(1), 293-304 (2014)

29. Pandey, S.K., Chaube, M.K.: Peristaltic flow of a micropolar fluid through a porous medium in the presence of an external magnetic field. Commun. Nonlinear Sci. Numer. Simul. 16, 3591-3601 (2011)

30. Rao, A.R., Mishra, M.: Peristaltic transport of power-law fluid in a porous tube. J. Non-Newton. Fluid Mech. 121, 163-174 (2004)

31. Shit, G.C., Roy, M., Ng, E.Y.K.: Effect of induced magnetic field on peristaltic flow of a micropolar fluid in an asymmetric channel. Int. J. Numer. Methods Biomed. Eng. 26(11), 1380-1403 (2010)

32. Shit, G.C., Ranjit, N.K., Sinha, A., Roy, M.: Effect of induced magnetic field on peristaltic transport of a micropolar fluid in the presence of slip velocity. Int. J. Appl. Math. Mech. 10(6), 81-107 (2014)

33. shit, G.C., Roy. M.: Hydromagnetic effect on inclined peristaltic flow of a couple stress fluid. Alex. Eng. J. (2014). doi:10.1016/j.aej.2014.07.007

34. Sobh, A.M., Mady, H.H.: Peristaltic flow through porous medium in a non-uniform channel. J. Appl. Sci. 8(6), 1085-1090 (2008)

35. Srivastava, L.M., Srivastava, V.P.: Peristaltic transport of power-law fluid: application to the ductus of efferentes of the reproductive tract. Rheol. Acta 27, 428-433 (1988)

36. Srivastava, L.M., Srivastava, V.P.: Peristaltic transport of a particle-fluid suspension. Trans. ASME J. Biomech. Eng. 111, 157-165 (1989)

37. Takabatake, S., Ayukava, K.: Numerical study of two dimensional peristaltic flows. J. Fluid Mech. 122, 439-465 (1982)

38. Vishnyakov, V.I., Pavlov, K.B.: Peristaltic flow of a conductive liquid in a transverse magnetic field. Magnetohydrodyanamics 8, 174-178 (1972)

39. Wang, Y., Ali, N., Hayat, T., Oberlack, M.: Peristaltic motion of a magnetohydrodynamic micropolar fluid in a tube. Appl. Math. Model. 35, 3737-3750 (2011) 